\title{
A Glimpse on Melanoma - Risk Factors and Treatment Soumya $D^{*}$
}

Department of Microbiology, Chaitanya Postgraduate College, Kakatiya University, Warangal, India

\begin{abstract}
Melanoma is the most dreadful form of skin cancer that begins in melanocytes. It is the leading cause of death from skin disease. It can occur in any part of the body that contains melanocytes. Several internal and external risk factors of the body may contribute to the development of many cancer melanomas such as unexpected exposure to UV radiation, weakened immune system, family history etc. Melanoma diagnosed at early stages is typically cured by surgical excision, but advanced stages are often deadly. Cancer stem cells (CSC) are recently proposed to be the cancer initiating cells responsible for tumorigenesis and contribute to cancer resistance. In the present review I have discussed about melanoma incidence, detection with various modes of treatment.
\end{abstract}

Keywords: Melanoma; Melanocytes; Skin cancer; Signs and Symptoms; Risk factors; Detection of melanoma; Therapies for treatment

Abbreviations: MM: Malignant melanoma; QOL: Quality of life; UV: Ultraviolet; ART: Adaptive radiotherapy; TRT: Targeted internal radionuclide therapy TSC: tumor stem cells; CSC: Cancer stem cells; BCG: Bacillus Calmette-Guerin; MSCs: mesenchymal stem cells; ABC: ATP binding cassette

\section{Introduction}

Cancer is the condition where cells are marked by their unregulated proliferation [1]. It is the major cause of death in the world after cardiovascular diseases [2]. It generally develops when normal cells in a particular part of the body begin to grow out of control. Tumor consists of numerous cancer cells, the size and shape of a tumor may not be an accurate measure as it is an abnormal growth [3]. The increase in the tumor size also increases the risk of complications and chance of reoccurrence [4].

There are different types of cancers; all types of cancer cells continue to grow abnormally. Some types of cancer cells often travel to other parts of the body through blood circulation or lymph vessels (metastasis), where they begin to grow.

Human skin regulates heat and water loss from the body [5], while preventing the access of deadly chemicals or microorganisms. Skin of healthy individual comprises of three main layers: subcutaneous tissue, underlying dermis and cellular epidermis [6]. Skin is also affected by psoriasis [7], Kaposi's sarcoma [8] linked with inflammation, and angiogenesis and immunosuppression.

When an injury occurs, the skin repairs itself through the proliferation and growth of dermal and epidermal cells [9]. But in deep skin lesions, destruction of the dermal and epidermal layers may take place thus the regeneration process is slowed down and complications arise. The first step for production of reconstructed skin involves the proliferation of dermal (fibroblasts) and epidermal cells (keratinocytes and melanocytes). Melanomas are usually an irregular pigmented lesion of varying size, but appearance may be atypical [10]. In case of metabolism of cancer cells and also for cell proliferation mitochondria plays a vital role [11].

In the treatment of large skin injuries, a higher number of applicable fibroblasts and keratinocytes are required [12]. In case of human keratinocytes media formulations, factors such as insulin, transferrin and lipid emulsion (containing cholesterol, $\alpha$-tocopherol acetate, fatty acids, Tween 80, and Pluronic F-68) should be assessed [13].

\section{Melanoma}

Melanoma is the alarming and most aggressive type of skin cancer which begins in melanocytes. Melanocytes are the cells that make the dark pigment called melanin which is responsible for the colour of skin. Melanoma may not be confined only to skin but also spreads to other pigmented tissues of the body, such as eye, bowel, and the intestines [14]. If it is confined to the skin, it can often be cured by surgery. However if it has spread, melanoma is usually incurable because it does not respond to most treatments. Recently clinicians have been trying a combination of chemotherapy and immunotherapy in the hope of improving the outcome [15].

The progression of Cancer depends upon physiological changes [16]. Metastatic melanoma may cause nonspecific paraneoplastic symptoms, including loss of appetite, nausea, vomiting and fatigue [17]. Metastasis of early melanoma is possible, but relatively rare: less than a fifth of melanomas diagnosed early become metastatic. Brain metastases are particularly common in patients with metastatic melanoma [18].

Though the incidence of Melanoma is still increasing, the mortality rate has remained unchanged. Alteration of Lymph node is the only most important prognostic factor for stage I/II melanoma patients. Right now, the standard of care with regard to the staging of these patients is the surgical sentinel node procedure. Ultrasound is not customary for the diagnostic work-up of primary melanomas [19]. In light of advanced and promising adjuvant therapies, the need for ultrasound staging might increase at speed. The advanced stages of melanoma are inevitably resistant to common therapeutic agents.

*Corresponding author: Soumya D, Department Of Microbiology, Chaitanya Postgraduate College, Kakatiya University, Warangal, India. E-mail: soumya. naidu22@gmail.com

Received July 12, 2011; Accepted September 24, 2011; Published September 26, 2011

Citation: Soumya D (2011) A Glimpse on Melanoma - Risk Factors and Treatment J Cancer Sci Ther S17. doi:10.4172/1948-5956.S17-004

Copyright: (๑) 2011 Soumya D. This is an open-access article distributed under the terms of the Creative Commons Attribution License, which permits unrestricted use, distribution, and reproduction in any medium, provided the original author and source are credited. 
In particular, epigenetic approach [20] might open interesting perspectives in combination with conventional ones. In my view, further investigations are needed to be conscious of the toxicity of these treatments in order to promote the survival of melanoma patients.

The therapeutic options remain confined for advanced MM, and those directed to the neoplastic cells have not brought major survival advantage so far. Immunotherapy is another focused choice [21]. By incorporating active and passive target techniques we can improve the efficiency of imaging techniques, allowing for earlier diagnosis and treatment of lethal conditions such as malignant growth [22]. Malignant tumors such as breast cancer, gastric cancer may be metastatic cause and basal cell carcinomas, melanoma, and angiosarcoma can be localized cause [23].

There are various types of Melanoma, which include the following.

- Superficial spreading melanoma: This type of melanoma is more commonly observed in the locations such as the legs of women and the backs of men. These are often barely raised and have a variety of colors.

- Nodular melanoma: This type of melanoma is known to progress faster and more likely to spread. Approximately $20 \%$ of melanomas take their origin as deeper, blue-black to purplish lumps.

- Lentigo maligna: This type of melanoma tends to occur on locations like the face, which are exposed to the sun regularly. They appear like a large, irregularly shaped or colored freckle and develop gradually. It takes many years to evolve into a fatal melanoma.

- Acral-lentiginous melanoma: This is the more common type of melanoma in nonwhites. Often they appear as irregular enlarging black flat spot (macule) on the palm and sole, rarely on a mucosal surface, such as the vulva or vagina.

Other occasional forms of melanoma may occur under the nails, on the palms and soles, in the eye, or at times even in the internal parts of the body.

\section{Risk Factors of Melanoma}

Many human organs can take several years to turn cancerous and have many causative factors. In industrialized nations cancer is more common, but there has been a growth in cancer rates in developing countries also, as these nations adopt the diet and lifestyle habits of industrialized countries [24].

Several internal and external factors of the body may contribute to the advancement of many types of cancer. Also hormonal influences may be responsible for the carcinogenic risk [25]. With reference to the role of immune system in cancer, psychosocial influences on immune function provide a mechanism of association between psychosocial factors (like interpersonal aggression) and prediction of cancer [26]. The actual cause of melanoma is not known but the risk factors that increase development of the disease are known. Risk factors for melanoma include:

- Unprotected exposure to UV radiation from natural sunlight or artificial sources, such as tanning beds/lamps

- Serious blistering sunburns
- Having fair skin and also having naturally red hair

- Family or personal history of melanoma

- Bearing many suspicious moles

- Weakened immune system

Other risk factors for melanoma include age, gender. Gender has been shown to be an essential and independent forecaster of clinical outcome and survival in cutaneous melanoma, with premenopausal females (but not women older than 60 years) experiencing an improved prognosis [27].

Xeroderma pigmentosum, Giant congenital melanocytic nevi, Dysplastic nervous syndrome, atypical nevi, many acquired, melanocytic nevi and immunosuppression are other risk factors. The absolute treatment is with surgical excision. Adjuvant therapies such as chemotherapy, immunotherapy, and radiation therapy can be used in advanced stages [28]. The occurrence of melanoma is increasing worldwide, with a growing fraction of patients with advanced disease for which diagnosis remain standing [29]. Therapeutic options are limited despite advances in immunotherapy and targeted therapy.

Signs and symptoms: Early signs of melanoma are changes to the shape or color of existing moles. At later stages, the mole may itch, ulcerate or bleed. Early signs of melanoma include:

- Irregularity of mole

- Blurred or ragged edges

- Alteration in the pigmentation of mole.

- Increase in the size and shape of mole

- Itching or tenderness of mole

Later symptoms include: pain in mole, swelling of lymph node, weight loss, chronic cough, gray skin, seizures etc.

\section{Preventive Measures}

The earlier the melanoma is diagnosed and treated, the better chance the patient has of surviving. But when left untreated, it spreads to other organs in the body. The high rate of metastasis is the main reason melanoma is considered the deadliest skin cancer. As "Prevention Is Better than Cure" measures should be followed to prevent occurrence of melanoma. Not all melanomas can be prevented, but can reduce the risk.

- The best way to reduce the risk of melanoma is to limit exposure to strong sunlight and other sources of UV light. And also tanning beds and sunlamps

- Protecting skin with clothing

- Usage of sunscreen

Vitamin D has many health benefits and it may aid to lower the risk for some cancers. The major source of vitamin D synthesis in humans is through the skin exposed to solar UV radiation [30]. If possible, it is better to obtain vitamin $\mathrm{D}$ from diet rather than from the sun. Abnormal or suspicious moles have to be checked and removed. And routine follow-up is to be carried out [31]. If the moles are altering into suspicious skin lesions, medical attention should be accomplished in a right away [32]. Also skin, scalp, feet, nails, and genital area should surely be examined as melanoma can appear on parts of the body that 
people do not consider checking. Genetic counseling and testing should be as a measure for reducing the risk of melanoma as gene mutation increases the risk of melanoma.

Detection of melanoma: Unlike infectious diseases, cancers arise from our own cells towards which the immune system has been tolerized. Therefore, effective immune responses against cancer would be challenging than it is against infectious diseases [33]. Generally Melanoma [34] diagnosed at early stages is cured by surgical excision alone [35], But in advanced stages it is often fatal [36]. It is crucial to detect melanoma at early stages as surgical excision is the only means of life saving. However, analyzing benign pigmented lesions and early melanomas can be challenging and hence there is a need for reliable markers that would boost in the diagnosis [37]. Still it is unexplored whether screening for early detection of melanoma enhances survival [38].

\section{Treatment}

Current advances in the diagnosis and treatment of cancer have led to an increase in the survival and hence, there is a greater emphasis on quality beside quantity of survival [39]. Effective treatment for advanced melanoma is still lacking. And hence it is better to limit exposure to ultraviolet radiation which remains the most effective way to reduce the risk of melanoma [40]. In-vivo testing devices include the devices or sensors that can be used to detect cancer [41].

Therapies for Treatment: Traditional approaches for the encounter of cancer include surgery, chemotherapy, and radiation therapy [42]. A new idea in cancer therapy is to enhance the selectivity of cytotoxic or cell-killing molecules by directly delivering them to cancer cells via the targeting of antigens that are unique to or highly expressed on the surface of cancer cells [43]. Fibroblast-derived cytokines have an impact on the immune system in tumor tissue [44].

The treatment becomes more complex when melanoma grows deeper into the skin or spreads. Surgeries may be needed to treat. Other treatments for melanoma include:

- Lymphadenectomy: This is the surgery which involves the removal of lymph nodes.

- Immunotherapy: This therapy aids the patient's immune system fight the cancer e.g.: interferon injections.

- Chemotherapy: Therapy that kills the cancer cells using chemicals.

- Radiation therapy: Radiations are used to kill the cancerous cells in this therapy.

Cancer treatment involving chemotherapy has toxic side effects; hence drug dosage given to patient should be limited. As a result, all of the tumor tissue may not be exposed to a lethal dose of the drug. The use of nanocarriers like liposomes and micelles can enhance the pharmacological properties of conventional chemotherapeutics [45]. Chemotherapy is associated with higher toxicity and lower quality of life (QOL) [46]. The secondary metabolites from microbes play a crucial role in generating chemotherapeutics [47]. It is known that different microorganisms are the source for various anticancer molecules [48]. A combination of treatment with radiotherapy and chemotherapy, or surgery can improve the prognosis of patients [49].

Many malignant tumours are hypoxic in tumor regions. This is clinically important as tumor hypoxia may increase resistance to radiation therapy and also to some forms of chemotherapy [50]. HIF$1 \mathrm{a}$ is involved in inflammatory pathways and angiogenesis and provides a therapeutic target. It also acts as a clinical biochemical marker for diagnosis of cancer [51].

An efficient and alternative to the current therapies for disseminated melanoma treatment is Targeted internal radionuclide therapy (TRT) [52]. Adaptive radiotherapy (ART) is a feedback method of treatment that optimizes according to patient-specific information measured during the course of treatment [53]. Cutaneous metastases from carcinoma are relatively uncommon in clinical practices when compared to metastases in various other organs of the body [54]. In the effective method of treatment for the metastatic melanoma cells to various other organs, Yttrium-90 microspheres radioembolization can be used [55]. Several toxicities and complications arise with radioembolization [56]. Epigenetic cancer therapy is becoming one of the most widely studied therapeutic agents for a vast array of diseases, including cancer [57].

The recent targeted biologic therapies will block essential functions of cancer cells and tumour stroma. A growing number of therapies, alone or in combination with other therapies (chemotherapy, hormone therapy, radiotherapy), will be a better adaptation of treatment to patients and disease characteristics [58]. Photo dynamic therapy [59] which was first applied in 1970s is another mode of control and treatment of melanoma cancers .It would be effective in combination with conventional methods. Tissue-engineered skin or Bioengineered skin can be used as an alternative to add various other cell types (keratinocytes, melanocytes, adipocytes, endothelial and immunological cells, etc.) according to needs [60].

In case of defective expression, DNA repair genes may affect the status of tumor DNA repair causing resistance to therapy, and affect the outcome of cancer and survival of patients. DNA repair enzymes and DNA repair inhibitors [61] are emerging as therapeutics in cancer diagnosis and development of cancer biomarkers to fight against cancer.

Anti-cancer drug -resistance could be developed as a strategy for research based on the evolution of drug resistance in malignant tissues [62].

Vaccines: Vaccines remain a promising but experimental treatment for melanoma [63]. Cancer vaccines, activating the immune system against specific antigens, have demonstrated clinical benefit and an increasing number are now in development [64]. Vaccines are a novel means in the treatment of melanoma. They are unique in their potential ability to induce long-lasting immune responses that can selectively attack and kill tumor cells. At present a variety of vaccines are being tested in clinical trials. Melanoma vaccines have minimal toxicity and appear to be much safer than traditional therapy with interferon that causes serious toxicity in up to two thirds of patients. Vaccines can induce antibody and/or cellular immune responses. Early trials of BCG vaccine-based immunotherapy for melanoma consistently show a trend toward improved clinical results in patients treated with BCG compared with observation alone [65].

TLR (Toll-like receptors) are exploring to be used in combination with cancer vaccines and hold significant promise for enhancing the efficacy of immunotherapeutics for promoting tumor-specific immunity [66].

Immunoglobulins with undefined specificity [67] are required 
for the development and viability of cancer cells from various tissue origins in human. Therefore, target-specific anti-cancer drugs can be developed, if we can identify unique biomarkers that can differentiate cancer cell-expressed immunoglobulins from those originated from normal B cells.

Palliative care: This care does not treat the cancer but can relieve symptoms and improve a patient's quality of life. When melanoma spreads, palliative care can aid in controlling the pain and other symptoms.

Drugs: Melanoma is a kind of cancer which represents about $1 \%$ of all tumors and is responsible for $75 \%$ of skin cancer deaths. Dacarbazine is currently the only approved chemotherapy drug for the standard treatment of metastatic melanoma stage IV, but its therapeutic response is not satisfactory and only $15-20 \%$ of patients with favorable prognosis have increased survival rate [68]

- Melanoma can become a model in cancer therapy. At present there are two drugs namely ipilimumab and vemurafinib with different characteristics and effectiveness. Vemurafinib acts immediately, with a median latency of 6-7 months before developing resistance, ipilimumab with a slower (months) but more lasting (years) action [69].

- For the future success of melanoma therapeutics, the use of combination treatments in which different regulatory pathways or the immunological response are targeted seems to be a promising tool [70].

- Clinical trials and preclinical testing are the basis for the standard approach to evaluate novel compounds for cancer treatment after drug synthesis or discovery [71].

- A new technology of novel double lumen catheter for drug delivery in skin is on its trails for its applications in humans [72]. It aids in preventing infections.

Natural sources i.e. honey can be used against cancer and invasive infections. Many anticancer compounds obtained from Ziziphus sp. such as betulinic acid are selective inhibitor drugs for the growth of human melanoma [73] cell lines by causing apoptosis.

Cancer stem cells (CSC) are the cancer initiating cells responsible for tumorigenesis and contribute to cancer resistance. Tumor stem cells may display ATP binding cassette (ABC) transporters which have been reported as TSC markers in melanoma \& osteosarcoma. For instance $\mathrm{ABCB} 5$ has been reported as a marker for a subset of CD133+ melanoma stem cells [74]. Cancer stem cells can arise from mesenchymal stem cells (MSCs) recruited to the tumor microenvironment [75]. MSCs [76] take their origin from fetus mesoderm layer and in the adult reside in different tissues like bone marrow stem cells (BMSCs), dermal stem cells etc. Human MSCs in the absence of ascorbic acid produce minimal amounts of collagen, which in turn aids in the inhibition of proliferation [77]. Tissue-specific stem cells could be the source of the original tumor. The existence of cancer stem cells is mostly accepted by the scientific community [78]. The identification of cancer stem cell with specific maintenance marker and its role in the differentiation would provide critical information for advancing towards the longterm goal of developing novel therapeutic strategies and will reduce the incidence of tumor recurrence for cancer patients [79]. Haematopoietic stem cells were widely used in transplantation experiments, especially in the treating leukemia and other cancers [80].
Melanoma cell line was cultivated in soft agar to check-out the effect of sugarcane cystatin in the anchorage-independent growth Sugarcane cystatin CaneCPI-4 inhibits melanoma development in vivo by angiogenesis disruption and prevention of melanoma invasion, migration and anchorage-independent growth. CaneCPI-4 efficiently inhibited melanoma cell invasion in vitro using a Transwell invasion assay [81]. The current dogma in the field of cancer is that cell lines contain at least two subpopulations of cells: cancer stem cells and noncancer stem cells [82]. Response rate, relapse free survival, disease stabilization rate overall survival, progression free survival in different phases are considered to be the principal primary endpoints used in advanced melanoma [83].

Proteomics has been employed recently to identify new disease related biomarkers for cancer diagnosis and development of targeted treatment [84]. Research studies are going for the employment of novel biomarkers in the diagnosis of cancers [85].

The ultimate goal of cancer therapy is to develop therapy with higher specificity for target tissues or cells. Certainly in the case of conventional chemotherapy agents such as doxorubicin, cisplatin etc., systemic administration results in cytotoxic effects not only into tumor cells, but also in healthy tissues.

\section{Emergence of Nanomedicine}

Nanomedicine has arrived into the cancer diagnostics and therapeutics [86]. Nanotechnologies cite a great opportunity to develop new products against cancer [87]. Nanotechnology definitely promises to serve as drug delivery carriers for the more challenging traditional drugs used for the treatment and management of chronic diseases such as cancer [88]. Nanoparticles or nanoporous particles functionalized with organic groups can be used as biomarkers, tracer, and drug delivery systems with much functionality for the treatment of cancer [89].

Using targeted nanoparticles to deliver chemotherapeutic agents in cancer therapy offers many advantages to improve drug or gene delivery thus to overcome many problems associated with conventional chemotherapy [90]. Liposomal nanoparticle therapeutics containing cytotoxic factors may provide the base for potentially more effective and less toxic anti-cancer treatment strategies due to their improved pharmacokinetics, reduced systemic toxicity, and increased intratumoral or intracellular delivery [91]. From the past few years there has been a growing attentiveness by various scientists to employ dendrimers for targeted delivery in cancer therapeutics [92].

\section{Conclusion}

In the present article I have discussed risk factors and various therapies for the treatment of melanoma. The use of combination treatments in which different regulatory pathways or the immunological response are targeted seems to be a promising tool for the future success of melanoma therapeutics. The Ultimate goal of cancer therapy is to develop therapy with higher specificity for target tissues or cells. Vaccines are a novel approach in the treatment of melanoma. Also Nanotechnology and Nanomedicine is a promising tool for the treatment and management of chronic diseases such as cancer. The identification of cancer stem cells with specific marker would provide critical information for advancing towards the long-term goal of developing novel therapeutics and will reduce the incidence of tumor recurrence for cancer patients. Several researches are on set and soon we will find the advanced and best mode of treatment. 


\section{Acknowledgement}

I would like to thank my friends for their support in writing the review article.

\section{References}

1. Sai YRKM, Dattatreya A, Anand SY, Mahalakshmi D (2011) Biomarkers and their Role in Premonition, Interpretation and Treatment of Cancer. J Cancer Sci Ther R1: 002

2. Sudhakar A (2009) History of Cancer, Ancient and Modern Treatment Methods. J Cancer Sci Ther 1: i-iv.

3. Retsky M (2011) Metronomic Chemotherapy was Originally Designed and firs used in 1994 for Early Stage Cancer - why is it Taking so Long to Proceed? J Bioequiv Availab 3: 00i-Oiv.

4. Osada S, Imai H, Sasaki Y, Yoshida K (2011) Cryoablation-Induced AntiCancer Immune Reaction. J Clinic Experiment Ophthalmol 2:151.

5. Williams A (2003) Transdermal and Topical Drug Delivery: from Theory to Clinical Practice in The Annals of Pharmacotherapy. Pharmaceutical Press, London, England.

6. McGrath JA, Eady RAJ, Pope FM (2004) Anatomy and Organization of Human skin. Rook's Textbook of Dermatology, Seventh Edition, Blackwell Publishing, USA 3.1-3.6.

7. Canavese M, Peric M, Dombrowski Y, Koglin S, Ruzicka T, et al. (2011) VEGF Induces IL-23 Expression in Keratinocytes through p38 Signaling. J Clin Exp Dermatol Res S2: 002

8. Essadi I, Sbitti Y, Ichou M, Errihani H (2011) The Role of Chemotherapy in the Treatment of Kaposi's Sarcoma. J Cancer Sci Ther 3: 145-148.

9. Souto LR, Vassallo J, Rehder J, Pinto GA, Puzzi MB (2009) Immunoarchitectural characterization of a human skin model reconstructed in vitro. Sao Paulo Med J 127: 28-33.

10. Buis PAJ, van Kemenade FJ, Frijling BD, van Diest PJ (2011) Skin Melanomas Excised by General Practitioners: More Often Unsuspected, of Nodular Type and Less of Often Radically Excised Than Those Excised in an Academic Setting. J Clin Exp Dermatol Res 2: 125

11. Yoshida $Y$, Hoshino S, Izumi H, Kohno K, Yamashita Y (2011) New Roles of Mitochondrial Transcription Factor A in Cancer. J Physic Chem Biophysic 1: 101.

12. Mazlyzam AL, Aminuddinn BS, Saim L, Ruszymah BHI (2008) Human Serum is an Advantageous Supplement for Human Dermal Fibroblast Expansion: Clinical Implications for Tissue Engineering of Skin. Arch Med Res 39: 743-752.

13. Xavier Batista FR, Rehder J, Puzzi MB (2010) Evaluation of Culture Medium for Human Keratinocytes. J Stem Cell Res Ther 1: 101

14. http://www.cancer.gov/cancertopics/types/melanoma

15. Sasse AD, Sasse EC, Clark LGO, Ulloa L, Clark OAC (2007) Chemoimmunotherapy versus chemotherapy for metastatic malignant melanoma. Cochrane Database Syst Rev 1: 1-48.

16. Mi Z, Guo H, Markovic J, Kuo PC (2009) Characterization of Osteopontin Binding Kinetics In MDA-MB231 Breast and SK-Hep-1 Liver Cancer Cells. J Cancer Sci Ther 1: 047-051.

17. http://melanomawarningsigns.com/

18. Fiddler IJ (1995) Melanoma Metastasis. Cancer Control 2: 398-404.

19. Ulrich J, van Akkooi AJ, Eggermont AM, Voit C (2011) New developments in melanoma: utility of ultrasound imaging (initial staging, follow-up and preSLNB). Expert Rev Anticancer Ther 11: 1693-701.

20. Porta CL (2011) Melanoma and Epigenetic Treatment: Past and Future: Anticancer Agents Med Chem.

21. Pierard GE, Aubin F, Humbert P. Ipilimumab (2011) A Promising Immunotherapy with Increased Overall Survival in Metastatic Melanoma? Dermatol Res Pract Epub 2011.

22. Rosen JE, Yoffe S, Meerasa A, Verma M, Gu FX (2011) Nanotechnology and Diagnostic Imaging: New Advances in Contrast Agent Technology. J Nanomedic Nanotechnol 2: 115.

23. Kim SH, Go JW, Cho HK (2011) Ectopic Syringoma with Localized Alopecia in Axillary Region. J Clin Exp Dermatol Res 2: 116

24. Singh RK, Sudhakar A, Lokeshwar BL (2011) From Normal Cells to Malignancy:
Distinct Role of Pro-inflammatory Factors and Cellular Redox Mechanisms. J Cancer Sci Ther 3: 070-075.

25. Wesserling M, Drewa T (2010) Will In Vitro Tests Replace Animal Models in Experimental Oncology? J Tissue Sci Eng 2: 102e.

26. Skopec R (2011) Mechanism Linking Aggression Stress through Inflammation to Cancer. J Cancer Sci Ther 3: 134-139.

27. Dronca RS, Markovic SN, Holtan SG, Porrata LF (2011) Neuro-endocrineimmune Crosstalk and Implications for Cancer Therapy. J Cell Sci Ther 2: 102e.

28. Jen M, Murphy M, Grant Kels JM (2009) Childhood melanoma. Clinics in Dermatology 27: 529-536.

29. Garbe C, Eigentler TK, Keilholz U, Hauschild A, John MK (2010) Systematic Review of Medical Treatment in Melanoma: Current Status and Future Prospects. The Oncologist 16: 5-24.

30. Stajner I (2009) Cloudiness and Breast Cancer. J Cancer Sci Ther 1: 034-040.

31. Dupont C (2010) Malignant Melanoma Detection in Ireland. J Clin Exp Dermato Res 1: 109.

32. Jones N, Colver GB (2011) Skin Cancer Nurses - A Screening Role. J Clin Exp Dermatol Res 2: 130.

33. Manjili MH (2011) Therapeutic Cancer Vaccines. J Clin Cell Immunol 2: e101.

34. Williams LH, Shors AR, Barlow WE, Solomon C, White E (2011) Identifying Persons at Highest Risk of Melanoma Using Self-Assessed Risk Factors. J Clin Exp Dermatol Res 2: 129.

35. Coit DG, Andtbacka R, Bichakjian CK, Dilawari RA, Dimaio D, et al. (2009) Melanoma. J Natl Compr Canc Netw 7: 250-275.

36. Eggermont AM, Testori A, Marsden J, Hersey P, Quirt I, et al. (2009) Utility of adjuvant systemic therapy in melanoma. Ann Oncol 20 Suppl 6: 30-34.

37. Abaffy T, Möller M, Riemer DD, Milikowski C, DeFazio RA (2011) A Case Repor - Volatile Metabolomic Signature of Malignant Melanoma using Matching Skin as a Control . J Cancer Sci Ther 3: 140-144.

38. Wolff T, Tai E, Miller T (2009) Screening for skin cancer: an update of the evidence for the U.S. Preventive Services Task Force. Ann Intern Med 150 194-198.

39. Pakseresht S, Ingle GK, Garg S (2011) Quality of Life of Women with Breast Cancer at the Time of Diagnosis in New Delhi. J Cancer Sci Ther 3: 066-069.

40. Dellavalle R, Drake A, Graber M, Heilig L, Hester E, Johnson K, McNealy K, Schilling L (2005) Statins and fibrates for preventing melanoma. Cochrane Database of Systematic Reviews.

41. Vijaya Shanti B, Mrudula T,Naga Deepth CH, Sree VenkateshwarluY (2011) Novel Applications of Nanotechnology in Life Sciences. J Bioanal Biomed R1: 001

42. Rosarin FS, Mirunalini S (2011) Nobel Metallic Nanoparticles with Nove Biomedical Properties. J Bioanal Biomed 3: 085-091.

43. Lechleider R, Pastan I (2011) Advances in the Development of Anti-CD22 Immunotoxins Containing Pseudomonas Exotoxin for Treatment of Hematologic Malignancies. J Cancer Sci Ther 3: 050-052.

44. Ghodsizad A, Voelkel T, Moebius JM, Gregoric I, Bordel V, et al. (2010) Biological Similarities Between Mesenchymal Stem Cells (Mscs) and Fibroblasts. J Cytol Histol 1: 101

45. Khan DR (2010) The Use of Nanocarriers for Drug Delivery in Cancer Therapy. J Cancer Sci Ther 2: 058-062.

46. Jamil K, Kumar K, Fatima SH, Rabbani S, Kumar R, et al. (2009) Clinica Studies on Hormonal Status in Breast Cancer and its Impact on Quality of Life (QOL). J Cancer Sci Ther 1: 083-089.

47. Samuilov VD (2003) Microbial therapy of cancer: Induction of Apoptosis, Recombinant Vaccines and Inhibition of Angiogenesis. Biochemistry (Mosc) 68: 958-962.

48. Ramachandran S, Sarkar S, Mazumadar A, Mandal M (2011) Azurin Synthesis from Pseudomonas Aeruginosa MTCC 2453, Properties, Induction of Reactive Oxygen Species, and p53 Stimulated Apoptosis in Breast Carcinoma Cells. J Cancer Sci Ther 3: 104-111.

49. Naga Deepthi CH, VVL Pavan Kumar A, Rameshbabu, Indirapriyadarshin U (2011) Role of Tumor Suppressor Protein p53 in Apoptosis and Cancer Therapy. J Cancer Sci Ther R1:001. 
50. Song WZ, Wang Z, Li LW, Chen JH, Wang J (2010) Evaluation of Early Response to Neoadjuvant Chemotherapy in Breast Cancer Patients by 18F-FDG and 99mTc-HL91 Imaging. J Nucl Med Radiat Ther 1: 102.

51. Nagy MA (2011) HIF-1 is the Commander of Gateways to Cancer. J Cancer Sci Ther 3: 035-040.

52. Bonnet-Duquennoy M, Papon J, Mishellany F, Denoyer D, Labarre $\mathrm{P}$, et al. (2009) Promising Pre-clinical Validation of Targeted Radionuclide Therapy Using a [1311] Labelled lodoquinoxaline Derivative for an Effective Melanoma Treatment. J Canc Sci Ther 1: 001-007.

53. Wang CY, Lee TF, Fang CH (2009) 3D Volumetric Visualization with Automatic Rigid and Deformable Hybrid Image Registration for Adaptive Radiotherapy. J Cancer Sci Ther 1: 041-046.

54. Pruneddu S, Piras D, Wijesuriya N, Cerio R (2011) Crohn's Disease and Mucocutaneous Conditions. J Clin Exp Dermatol Res S3:001.

55. Ettorre GM, Vennarecci G, Santoro R, Miglioresi L, Lepiane P, et al. (2011) Experiences in Hepatic Surgery and Transplantation after Radioembolization. J Nucl Med Radiat Ther 2: 109.

56. Liu DM, Cade D, Klass D, Loh C, McWilliams JP, et al. (2011) Interventional Oncology - Avoiding Common Pitfalls to Reduce Toxicity in Hepatic Radioembolization. J Nucl Med Radiat Ther 2: 106.

57. de Almeida VR, Brunetto AL, Schwartsmann G, Roesler R, Abujamra AL (2011) De-mystifying the Epigenetic Free for All: Pharmacophore Modeling for Epigenetic Cancer Therapy. Pharm Anal Acta 2: 102e.

58. Bareggi Renato NV, Paola N (2010) New Targeted Therapies Against Breast Cancer. J Carcinogene Mutagene 1: 110.

59. Devidas M (2011) An Evidence Scoping Review of Photodynamic Therapy (PDT) in the Treatment of Cancers. J Anal Bioanal Techniques S1: 002.

60. Jean J, Garcia-Pérez ME, Pouliot R (2011) Bioengineered Skin: The SelfAssembly Approach. J Tissue Sci Eng S5: 001.

61. Dizdaroglu M, Jaruga P (2011) Oxidatively Induced DNA Damage and Cancer. J Mol Biomark Diagn S2: 002

62. Zhang Y (2011) New Concepts of Germline Gene -reactivated Cancer. Human Genet Embryol 1: e101.

63. Bystryn JC, Rudolph JL (2005), Emerging melanoma vaccines: Expert Opin Emerg Drugs, 10(2):393-402 PMID: 15934874

64. Sinnathamby G, Zerfass J, Hafner J, Block P, Nickens Z, et al. (2011) EDDR1 is a Potential Immunotherapeutic Antigen in Ovarian, Breast, and Prostate Cancer. J Clin Cell Immunol 2: 106

65. Stewart JH, Levine EA (2011) Role of bacillus Calmette-Guérin in the treatment of advanced melanoma: Expert Rev Anticancer Ther 11: 1671-1676.

66. Gregory SM, West JA, Damania B (2011) Cancer Vaccination, Will You Have To Pay The Toll? J Vaccines Vaccin S1: 001.

67. Lee G, Cheung AP, Ge B, Zhu M, Li PP, et al. (2010) Monoclonal Anti-idiotype Antibodies against Carbohydrate-associate Epitope for Anti- Cancer Vaccine Development. J Vaccines Vaccin 1: 106

68. Ferreira AK, Meneguelo R, Neto SC, Chierice GO, Maria DA (2011) Synthetic Phosphoethanolamine Induces Apoptosis Through Caspase-3 Pathway by Decreasing Expression of Bax/Bad Protein and Changes Cell Cycle in Melanoma. J Cancer Sci Ther 3: 053-059.

69. Ascierto PA (2011) The Future of Melanoma Therapy is the Combination Approach. J Mol Biomark Diagn 2: 102e.

70. Heneberg P (2011) Advances in Clinical Treatment of Malignant Melanoma: B-RAF Kinase Inhibition. Klin Onkol 24: 256-64.

71. Avramidis D, Cruz M, Sidén A, Tasat DR, Yakisich JS (2009) Regrowth Concentration Zero (RCO) as Complementary Endpoint Parameter to Evaluate Compound Candidates During Preclinical Drug Development for Cancer Treatment. J Cancer Sci Ther 1: 019-024.

72. Peramo A (2010) Novel Double Lumen Catheter for Drug Delivery at the SkinCatheter Interface. J Tissue Sci Eng 1:102.

73. El-Gendy MMA (2010) In vitro, Evaluation of Medicinal Activity of Egyptian Honey from Different Floral Sources as Anticancer and Antimycotic Infective Agents. J Microbial Biochem Technol 2: 118-123.

74. Shrihari TG (2011) Cancer Stem Cells - Therapeutic Boon! J Cancer Sci Ther 3: $197-200$.
75. Razmkhah M, Jaberipour M, Ghaderi A (2011) Chemokines and Chemokine Receptors Expression in the Adipose Derived Stem Cells (ASCs), Breast Tissues and in Peripheral Blood of Patients with Breast Cancer. J Carcinogene Mutagene 2: 120.

76. Silvani G, Galli D, Benedetti L, Ceccarelli G, Crosetto N, et al. (2011) Peculia Characteristics of Human Mesenchymal Stem Cell Clones Suitable as Tissue Engineering Models. J Tissue Sci Eng 2: 106.

77. Pei M, He F, Wei L (2010) Three-Dimensional Cell Expansion Substrate For Cartilage Tissue Engineering And Regeneration: A Comparison In Decellularized Matrix Deposited By Synovium-Derived Stem Cells And Chondrocytes. J Tissue Sci Eng 2: 104.

78. Rameshwar P (2011) Post-identification of Cancer Stem Cell: Ethical and Scientific Dilemmas in Therapeutic Development?.JStemCellResTher1:e101

79. Ponnusamy MP (2010) Stem Cell Research and Cancer Stem Cells. J Tissue Sci Eng 2: 104e.

80. Meregalli M, Farini A, Torrente $Y$ (2011) Mesenchymal Stem Cells as Muscle Reservoir. J Stem Cell Res Ther 1: 105.

81. Oliveira JP, Magliarelli HF, Pereira FV, Gianotti A, Soares-Costa A, et al. (2011) Sugarcane Cystatin CaneCPI-4 inhibits Melanoma Growth by Angiogenesis Disruption. J Cancer Sci Ther 3: 161-167.

82. Cruz M, Siden A, Tasat DR, Yakisich JS (2010) Are all Glioma Cells Cancer Stem Cells? J Cancer Sci Ther 2: 100-106.

83. Kiba T (2011) The Choice of the Endpoint to Assess the Efficacy or Effectiveness in Advanced or Metastatic Cancer Tumors. J Cancer Sci Ther 3: 154-157.

84. Lu M, Whitelegge JP, Whelan SA, He J, Saxton RE (2010) Hydrophobic Fractionation Enhances Novel Protein Detection by Mass Spectrometry in Triple Negative Breast Cancer. J Proteomics Bioinform 3: 029-038.

85. Tadashi K(2008) Cancer Proteomics for Biomarker Development. J Proteomics Bioinform 1: 477-484.

86. Suh KS, Tanaka T (2011) Nanomedicine in Cancer. Translational Medic 1:103e.

87. Caraglia M, Rosa GD, Abbruzzese A, Leonetti C (2011) Nanotechnologies: New Opportunities for Old Drugs. The Case of Aminobisphosphonates. J Nanomedic Biotherapeu Discover 1:103e.

88. Anwunobi AP, Emeje MO (2011) Recent Applications of Natural Polymers in Nanodrug Delivery. J Nanomedic Nanotechnol S4: 002.

89. Menaa B (2011) The Importance of Nanotechnology in Biomedical Sciences. J Biotechnol Biomaterial 1: 105e.

90. Nguyen KT (2011) Targeted Nanoparticles for Cancer Therapy: Promises and Challenges. J Nanomedic Nanotechnol 2:103e.

91. Dibirdik I, Yiv S, Qazi S, Uckun FM (2010) In vivo Anti-Cancer Activity of a Liposomal Nanoparticle Construct of Multifunctional Tyrosine Kinase Inhibito 4-(4'-Hydroxyphenyl)-Amino-6,7-Dimethoxyquinazoline. J Nanomedic Nanotechnolo 1: 101.

92. Bharali DJ, Khalil M, Gurbuz M, Simone TM, Mousa SA (2009) Nanoparticles and cancer therapy: A concise review with emphasis on dendrimers. International Journal of Nanomedicine 4:1-7. 